\title{
Mesons upon low-lying Dirac mode exclusion
}

\section{Denissenya*}

Institut für Physik, FB Theoretische Physik, Universität Graz

E-mail: mikhail.denissenya@uni-graz.at

L. Ya. Glozman

Institut für Physik, FB Theoretische Physik, Universität Graz

E-mail: leonid.glozman@uni-graz.at

\section{B. Lang}

Institut für Physik, FB Theoretische Physik, Universität Graz

E-mail: christian.lang@uni-graz.at

\begin{abstract}
We study the isoscalar and isovector $J=0,1$ mesons with the overlap operator within two flavour lattice QCD. After subtraction of the lowest-lying Dirac eigenmodes from the valence quark propagator all disconnected contributions vanish and all possible point-to-point $J=0$ correlators become identical, signaling a simultaneous restoration of both $S U(2)_{L} \times S U(2)_{R}$ and $U(1)_{A}$ symmetries. The ground states of the $\pi, \sigma, a_{0}, \eta$ mesons do not survive this truncation. All possible $J=1$ states have a very clean exponential decay and become degenerate, demonstrating a $S U(4)$ symmetry of a dynamical QCD-like string.
\end{abstract}

The 32nd International Symposium on Lattice Field Theory,

23-28 June, 2014

Columbia University New York, NY

${ }^{*}$ Speaker. 


\section{Introduction}

in our previous study [1] we discovered a degeneracy of all isovector mesons of $\operatorname{spin} J=1$, $\rho, \rho^{\prime}, a_{1}, b_{1}$, upon truncation of the quasi-zero modes from the valence quark propagators with the manifestly chirally-invariant overlap Dirac operator ( for a previous study with the chirally improved Dirac operator see Refs. [2,3]). The density of the quasi-zero modes is directly related to the quark condensate of the vacuum [4]. Via such a truncation we artificially restore ( "unbreak") the chiral symmetry. All $J=1$ states survive the unbreaking, because a very clean exponential decay of the correlators is seen. Surprisingly, not only a degeneracy within the chiral partners $\rho$ and $a_{1}$ has been observed, but actually a degeneracy of all four states. This degeneracy signals not only a simultaneous restoration of both $S U(2)_{L} \times S U(2)_{R}$ and $U(1)_{A}$ symmetries, but of some higher symmetry that includes $S U(2)_{L} \times S U(2)_{R} \times U(1)_{A}$ as a subgroup. This symmetry would require a degeneracy of all possible chiral multiplets with $J=1$ that contain both isoscalar and isovector mesons. Here we report our results on both the isoscalar and isovector mesons [5].

Our second aim is to investigate the $J=0$ correlators in both the isovector and isoscalar channels and clarify the fate of the $\pi, \sigma, a_{0}, \eta$ mesons upon unbreaking.

\section{Lattice setup}

Our ensemble includes 100 gauge configurations generated by JLQCD with $n_{f}=2$ dynamical overlap fermions at fixed topology $Q_{t o p}=0$ [6]. The lattice size is $16^{3} \times 32$, the lattice spacing $a \sim 0.12$ and the pion mass in this ensemble is $M_{\pi}=289(2) \mathrm{MeV}$.

\subsection{Quark propagators}

The quark propagators, obtained from JLQCD, consist of two parts. The contribution of the first 100 Dirac eigenmodes was computed exactly, and the effect of all higher-lying modes was taken into account via a stochastic estimate [7]. Our unbreaking procedure means consequently a removal of the first $k$ modes from the quark propagators:

$$
S_{k}(x, y)=\sum_{n=k+1}^{100} \frac{1}{\lambda_{n}} u_{n}(x) u_{n}^{\dagger}(y)+S_{\text {Stoch }}(x, y) .
$$

The resulting full $(k=0)$ and reduced $(k>0)$ quark propagators are then used in the construction of the meson correlation functions.

\subsection{Meson observables}

We use the standard variational approach [8-10]. Our basis of operators is enlarged by introducing an exponential type of smearing at source/sink. We construct the cross-correlation matrices

$$
C_{i j}(t)=\left\langle 0\left|\mathscr{O}_{i}(t) \mathscr{O}_{j}^{\dagger}(0)\right| 0\right\rangle
$$

with the size up to $10 \times 10$ with a subsequent solution of the generalized eigenvalue problem

$$
C(t) \vec{v}_{n}(t)=\tilde{\lambda}^{(n)}(t) C\left(t_{0}\right) \vec{v}_{n}(t) .
$$

The masses of the eigenstates are obtained by identifying the exponential decay of the eigenvalues $\tilde{\lambda}^{(n)}(t)$. Such states are extracted for each low-lying mode truncation level $k$ in a given quantum channel. 


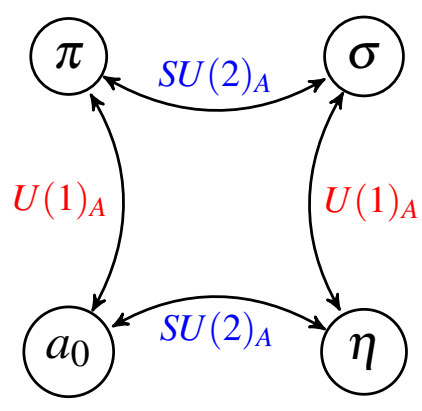

Figure 1: Symmetry relations between $J=0$ mesons
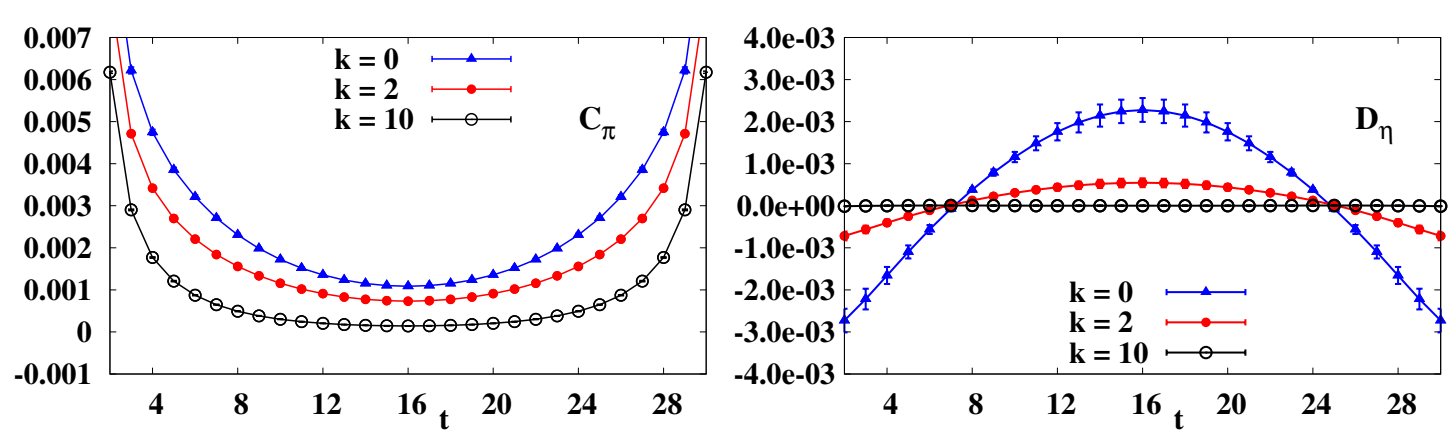

Figure 2: Connected (left) and disconnected (right) parts of the $\eta$ correlator upon the low-mode exclusion.

\section{Results}

\section{1 $J=0$ mesons}

The $J=0$ operators and the corresponding representations of the chiral group are listed in Table 1 . They are connected by the symmetry transformations that are illustrated in Fig. 1 [11,12].

The isovector correlation functions contain only connected $(C)$ contributions. Both the connected and disconnected $(D)$ contributions are important for the isoscalar correlators. In our case we have two degenerate quark flavours, i.e., there is no distinction between the $u$ and $d$ quark propagators. Hence, the connected part of the $\sigma$ correlator is identical to the $a_{0}$ correlator, the same argument applies to $\eta$ and $\pi$ :

$$
F_{\eta(\sigma)}=C_{\pi\left(a_{0}\right)}+D_{\eta(\sigma)},
$$

where $F$ represents the full correlator with given quantum numbers.

In Fig. 2 we show the connected and disconnected contributions in the $\eta$ channel. In the untruncated case $k=0$ they are both equally important. After elimination of $k \sim 10$ lowest eigenmodes the disconnected contribution essentially vanishes.

Figure 3 shows $\pi, \sigma, a_{0}, \eta$ point-to-point correlators at different truncation levels $k$. The $S U(2)_{L} \times S U(2)_{R}$ and $U(1)_{A}$ symmetries are strongly broken at $k=0$, which makes all four pointto-point correlators very different.

Upon elimination of a small amount of lowest-lying Dirac eigenmodes all correlators become identical. We conclude that both $S U(2)_{L} \times S U(2)_{R}$ and $U(1)_{A}$ symmetries get simultaneously 

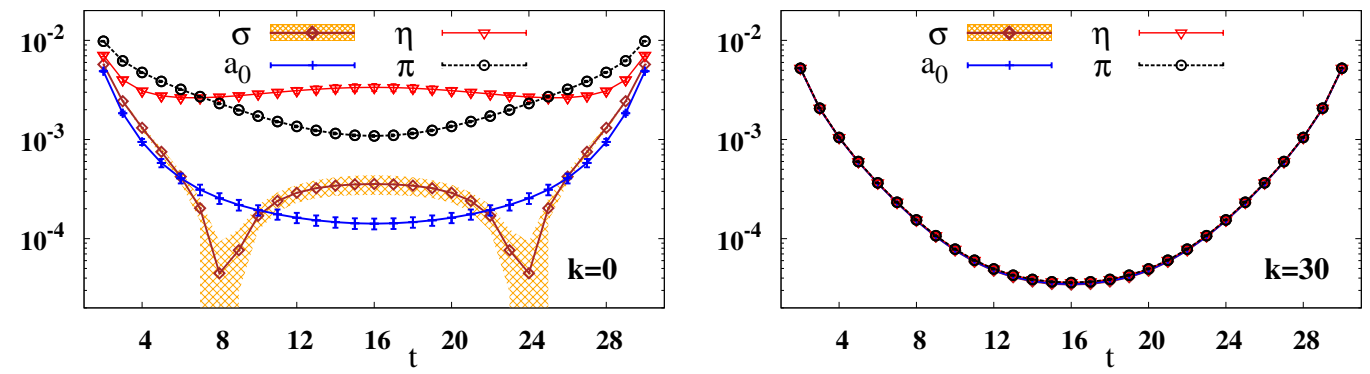

Figure 3: Two-point correlators of $\pi, \eta, \sigma, a_{0}$ mesons.
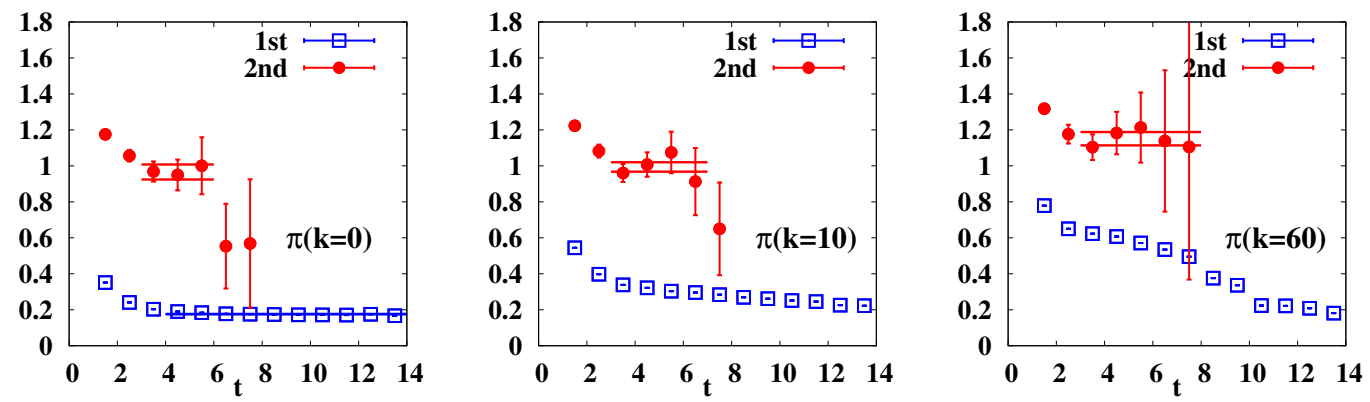

Figure 4: $\pi$ effective masses at $k=0,10,60$.

restored. The same quasi-zero modes are responsible for both symmetry breakings which is consistent with the instanton induced mechanism.

The next question is whether the $J=0$ ground states still persist after unbreaking. We consider the pseudo-scalar channel because the original $\pi$ states can be easily identified in the untruncated case. The effective mass for $\pi$ is shown on Fig. 4. For the untruncated case $k=0$ we see a very good effective mass plateau and consequently a clean exponential decay of the correlator. Upon exclusion of a small amount of the lowest-lying Dirac modes the correlation function decays with time no longer exponentially indicating the absence of a "physical" state. The unbreaking removes the pion from the physical spectrum, which is consistent with its Goldstone nature: The quasi-zero modes are crucially important for its existence. However, the first excited state of the pion might survive the truncation.

We have demonstrated earlier that both $S U(2)_{L} \times S U(2)_{R}$ and $U(1)_{A}$ symmetries get simultaneously restored upon unbreaking. Hence the ground states of $\sigma, a_{0}, \eta$ mesons should disappear

\begin{tabular}{|c|c|c|}
\hline \hline$I, J^{P C}$ & $\mathscr{O}$ & $R$ \\
\hline$\pi\left(1,0^{-+}\right)$ & $\bar{q} \gamma_{5} \frac{\tau}{2} q$ & $(1 / 2,1 / 2)_{a}$ \\
$\eta\left(0,0^{-+}\right)$ & $\bar{q} \gamma_{5} q$ & $(1 / 2,1 / 2)_{b}$ \\
$a_{0}\left(1,0^{++}\right)$ & $\bar{q} \frac{\tau}{2} q$ & $(1 / 2,1 / 2)_{b}$ \\
$\sigma\left(0,0^{++}\right)$ & $\bar{q} q$ & $(1 / 2,1 / 2)_{a}$ \\
\hline$\rho\left(1,1^{--}\right)$ & $\bar{q} \gamma_{i} \frac{\tau}{2} q$ & $(1,0) \oplus(0,1)$ \\
& $\bar{q} \gamma_{i} \gamma_{t} \frac{\tau}{2} q$ & $(1 / 2,1 / 2)_{b}$ \\
\hline$\omega\left(0,1^{--}\right)$ & $\bar{q} \gamma_{i} q$ & $(0,0)$ \\
& $\bar{q} \gamma_{i} \gamma_{t} q$ & $(1 / 2,1 / 2)_{a}$ \\
\hline$a_{1}\left(1,1^{++}\right)$ & $\bar{q} \gamma_{i} \gamma_{5} \frac{\tau}{2} q$ & $(1,0) \oplus(0,1)$ \\
$f_{1}\left(0,1^{++}\right)$ & $\bar{q} \gamma_{i} \gamma_{5} q$ & $(0,0)$ \\
$b_{1}\left(1,1^{+-}\right)$ & $\bar{q} \gamma_{i} \gamma_{j} \frac{\tau}{2} q$ & $(1 / 2,1 / 2)_{a}$ \\
$h_{1}\left(0,1^{+-}\right)$ & $\bar{q} \gamma_{i} \gamma_{j} q$ & $(1 / 2,1 / 2)_{b}$ \\
\hline
\end{tabular}

Table 1: $J=0$ and $J=1$ meson interpolating fields $\mathscr{O}: R$ denotes an index of the chiral multiplet within each $J$ [11]. 

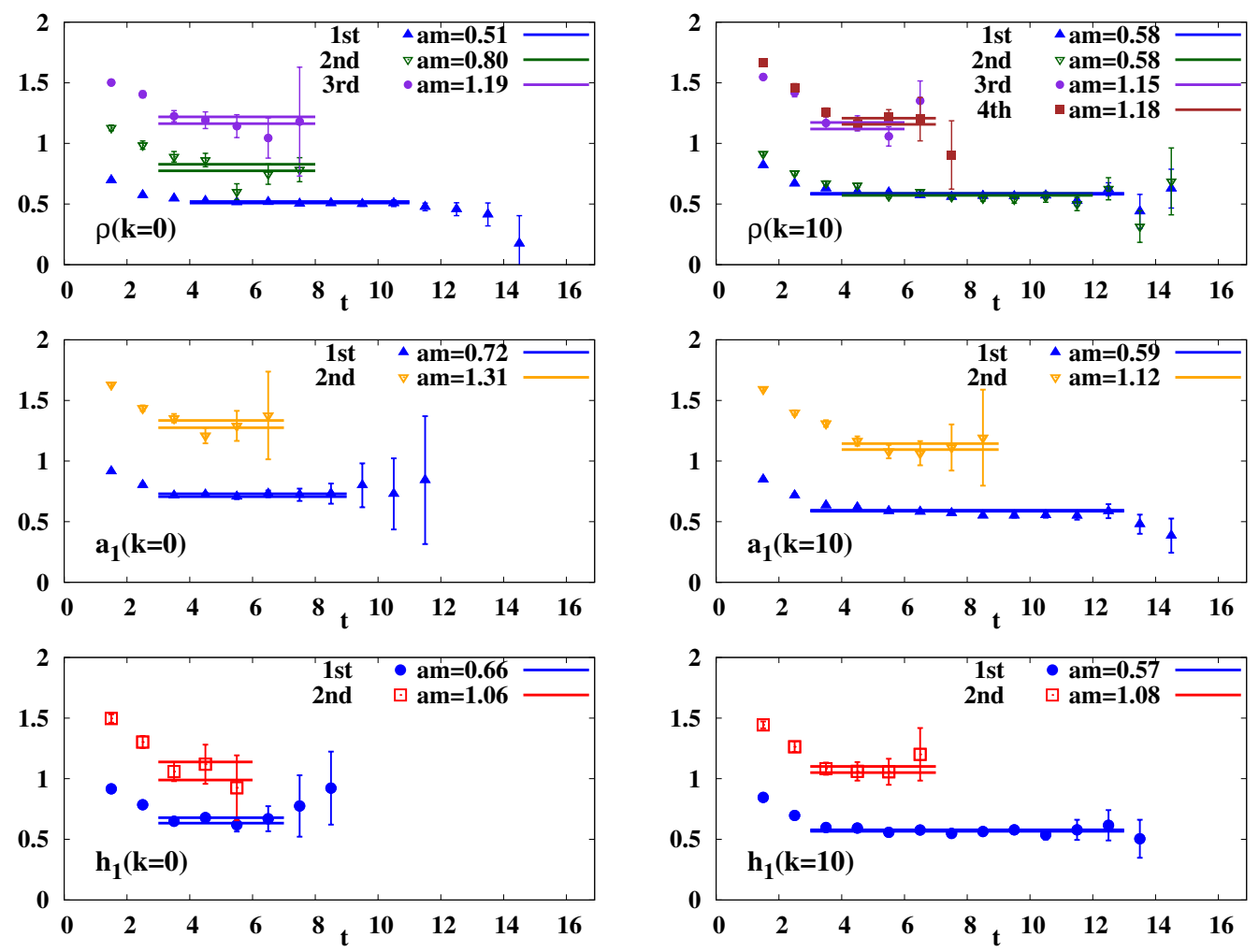

Figure 5: $\rho, a_{1}$ and $h_{1}$ effective masses at $\mathrm{k}=0,10$.

from the spectrum simultaneously with the pion. The opposite would contradict the restoration of both symmetries.

\section{2 $J=1$ mesons}

The interpolating fields and the respective chiral representations are given in Table 1. In contrast to the $J=0$ case the disconnected contributions to the isoscalar correlators are small compared to the connected ones. The interpretation is that the disconnected contributions are supported in the infrared by the instantons, i.e., by the 't Hooft vertex. This vertex is limited, however, to the $J=0$ channel. After subtraction of the lowest eigenmodes the small disconnected contributions in the $J=1$ case vanish.

All $J=1$ states survive the unbreaking, see Fig. 5: After elimination of the lowest-lying Dirac eigenmodes we observe a clean exponential decay of the corresponding eigenvalues. The evolution of the $J=1$ meson masses is shown on Fig. 6. All possible states get degenerate upon elimination of $\sim 10$ modes. This indicates some larger symmetry that includes $S U(2)_{L} \times S U(2)_{R} \times U(1)_{A}$ as a subgroup. This symmetry can be reconstructed and turns out to be a $S U(4)$ [13], mixing components of the fundamental four-component vector $\left(u_{L}, u_{R}, d_{L}, d_{R}\right)$. This symmetry is not a symmetry of the QCD Lagrangian but should be considered as an emergent symmetry that appears from the QCD dynamics upon elimination of the quasi-zero Dirac eigenmodes. It operates only for $J \geq 1$ states. From this symmetry it is possible to conclude that after truncation there is no color- 


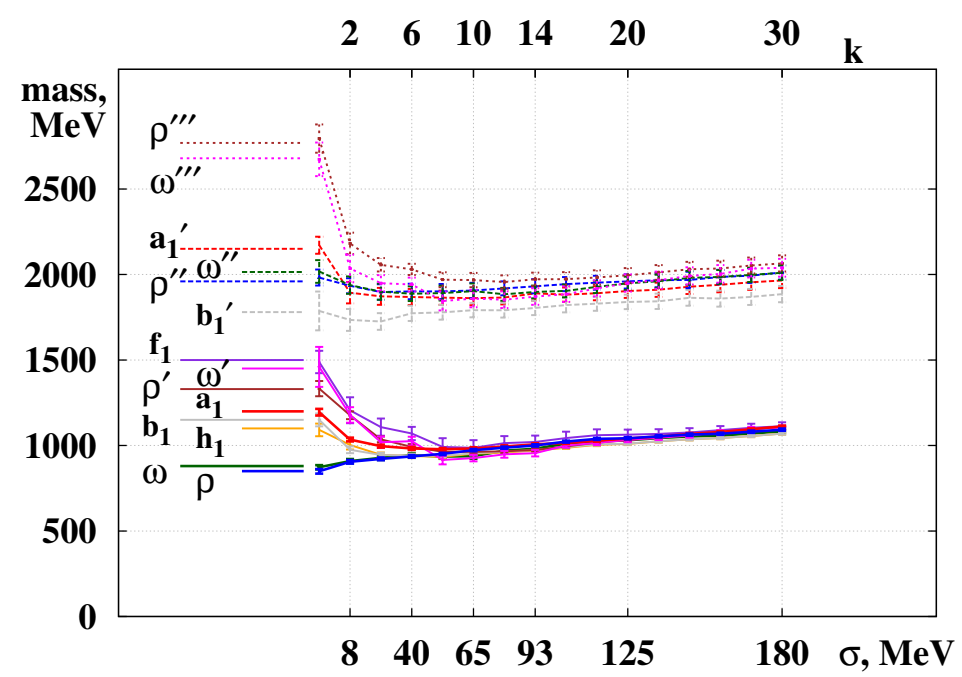

Figure 6: Mass evolution of $J=1$ mesons upon exclusion of the low-lying Dirac modes, $\sigma$ denotes the energy gap.

magnetic field in the system suggesting that we observe quantum levels of a dynamical QCD-like string.

\section{Acknowledgements}

We thank S. Aoki, S. Hashimoto and T. Kaneko for supplying us with the JLQCD gauge configurations and quark propagators and their help and hospitality. The calculations were performed on computing clusters of the University of Graz (NAWI Graz). Support from the Austrian Science Fund (FWF) through the grants DK W1203-N16 and P26627-N16 is acknowledged.

\section{References}

[1] M. Denissenya, L. Y. Glozman and C. B. Lang, Symmetries of mesons after unbreaking of chiral symmetry and their string interpretation, Phys. Rev. D 89, 077502 (2014);

[2] C. B. Lang and M. Schröck, Unbreaking chiral symmetry, Phys. Rev. D 84, 087704 (2011).

[3] L. Y. Glozman, C. B. Lang and M. Schröck, Symmetries of hadrons after unbreaking the chiral symmetry, Phys. Rev. D 86, 014507 (2012).

[4] T. Banks and A. Casher, Chiral Symmetry Breaking in Confining Theories, Nucl. Phys. B 169, 103 (1980).

[5] M. Denissenya, L. Y. Glozman and C. B. Lang, Isoscalar mesons upon unbreaking of chiral symmetry, arXiv:1410.8751.

[6] S. Aoki et al. [JLQCD Collaboration], Two-flavor QCD simulation with exact chiral symmetry, Phys. Rev. D 78, 014508 (2008).

[7] S. Aoki et al. Simulation of quantum chromodynamics on the lattice with exactly chiral lattice fermions, PTEP 2012, 01A106 (2012).

[8] C. Michael, Adjoint Sources in Lattice Gauge Theory, Nucl. Phys. B 259 (1985) 58. 
[9] M. Lüscher and U. Wolff, How to calculate the Elastic Scattering Matrix in 2-Dimensional QuantumField Theories by Numerical Simulation, Nucl. Phys. B 339 (1990) 222.

[10] B. Blossier, M. Della-Morte, G. von Hippel, T. Mendes, and R. Sommer, On the generalized eigenvalue method for energies and matrix elements in lattice field theory, JHEP 0904 (2009) 094.

[11] L. Y. Glozman, Chiral multiplets of excited mesons, Phys. Lett. B 587 (2004) 69.

[12] T. D. Cohen and X. -D. Ji, Chiral multiplets of hadron currents, Phys. Rev. D 55, 6870 (1997).

[13] L. Y. Glozman, SU(4) symmetry of the dynamical QCD string and genesis of hadron spectra, arXiv:1407.2798 [hep-ph]. 